\title{
Association of apelin and apelin receptor with the risk of coronary artery disease: a meta-analysis of observational studies
}

\author{
Tianbao Chen ${ }^{1}$, Bing Wu${ }^{1}$ and Rong Lin ${ }^{1}$ \\ ${ }^{1}$ Department of Cardiology, Fujian Medical University Affiliated the First Quanzhou Hospital, Quanzhou City, Fujian, People's \\ Republic of China \\ Correspondence to: Tianbao Chen, email: chentianbao@medmail.com.cn \\ Keywords: apelin-APLNR pathway, coronary artery disease, circulating apelin concentration, genetic polymorphism, meta-anal- \\ ysis \\ $\begin{array}{lll}\text { Received: March 08, } 2017 & \text { Accepted: April 04, } 2017 & \text { Published: April 21, } 2017\end{array}$
}

Copyright: Chen et al. This is an open-access article distributed under the terms of the Creative Commons Attribution License 3.0 (CC BY 3.0), which permits unrestricted use, distribution, and reproduction in any medium, provided the original author and source are credited.

\section{ABSTRACT}

It is well established that apelin-APLNR (apelin receptor) pathway plays a central role in cardiovascular system. In this meta-analysis, we summarized published results on circulating apelin concentration in association with coronary artery disease (CAD), apelin and APLNR genetic polymorphism(s) in predisposition to CAD risk and circulating apelin changes after surgical treatment for CAD. The results from 15 articles were pooled. Two authors independently took charge of literature search, article selection and information collection. Overall, circulating apelin concentration was significantly lower in CAD patients $(\mathrm{N}=1021)$ than in controls $(\mathrm{N}=654)$ (weighted mean difference [WMD]: $\mathbf{- 1 . 2 8 5} \mathbf{n g} / \mathbf{m L}, \mathbf{9 5} \%$ confidence interval [CI]: $\mathbf{- 1 . 7 9 0}$ to $-0.780, P<0001)$, with significant heterogeneity $\left(I^{2}=99.3 \%\right)$ but without publication bias. For the association of APLNR gene rs9943582 polymorphism with CAD (patients/ controls: $5975 / 4717$ ), the mutant $T$ allele was associated with a $5.2 \%$ increased risk relative to the wild C allele (odds ratio: $1.052,95 \% \mathrm{CI}: 0.990$ to $1.117, P=0.100$ ), without heterogeneity $\left(I^{2}=0.0 \%\right)$ or publication bias. Circulating apelin was increased significantly after surgical treatment for CAD $(\mathrm{N}=202)(W M D: 2.011 \mathrm{ng} / \mathrm{mL}, 95 \%$ CI: 0.541 to $3.481, P=0.007)$, with significant heterogeneity $\left(I^{2}=98.0 \%\right)$. Stratified analyses showed that circulating apelin was significantly reduced in studies with age- and sex-matched patients and controls (WMD: $-1.881 \mathrm{ng} / \mathrm{mL}, 95 \% \mathrm{CI}:-2.457$ to $-1.304, P<0.001$ ) and with total sample size $\geq 125$ (WMD: $-1.657 \mathrm{ng} / \mathrm{mL}, 95 \% \mathrm{CI}$ : -2.378 to $-0.936, P<0.001)$, relative to studies without matching reports and with total sample size $<125$. In brief, our results suggested that circulating apelin was a prominent athero-protective marker against the development of CAD.

\section{INTRODUCTION}

As released by the World Health Organization, coronary artery disease (CAD) is entitled as the world's biggest killer, contributing to approximately 8.76 million deaths in 2015, up from 5.74 million deaths in 1990 [1], and it will continue to be the single largest cause of disease burden in many countries worldwide by the year 2020 [2]. Several risk factors have been proposed to play a part in the development of CAD, such as age, sex, smoking, hypertension, diabetes mellitus and high-density lipoprotein cholesterol [3, 4]. However, conventional risk profiles cannot fully account for the process of coronary artery atherosclerosis, the underlying mechanism of CAD [5]. Recent years have come with major achievements in identifying a large panel of novel biomarkers $[6,7]$, such as tenascin-C [8] and LRP8 [9], whereas current prospects are far from encouraging, with no consensus on their medical implications. It is undoubtedly necessary to unfold a more comprehensive picture for certain promising markers affecting CAD based on the literature.

In the present study, we were intended to focus on a promising pathway containing apelin and apelin receptor (APLNR). Apelin, a peptide isolated from bovine stomach extracts, is the endogenous ligand for orphan receptor APLNR [10]. The biological plausibility of apelin-APLNR 
pathway in cardiovascular system is obvious, echoing some observations from clinical, epidemiologic, genetic, in-vivo and in-vitro studies. For instance, Japp et al in a clinical trial found that acute apelin administration in humans can evoke peripheral and coronary vasodilatation and raise cardiac output [11]. In a mouse model, apelin administration was found to be able to ameliorate high fat diet-induced cardiac hypertrophy and contractile dysfunction [12]. Several epidemiologic studies in humans have proposed circulating apelin as a promising predictor for CAD [13-17]; however, a systematic investigation is currently lacking. It is reasonable to hypothesize that apelin-APLNR pathway plays a central role in cardiovascular system. To fill this gap of knowledge, we summarized the results from published articles on the difference in circulating apelin concentration between CAD patients and controls through a comprehensive metaanalysis. Meanwhile, we summarized the results on apelin and $A P L N R$ genetic polymorphisms in predisposition to CAD risk. What's more, circulating apelin changes after surgical treatment for CAD were also summarized to seek additional supporting evidence.

\section{RESULTS}

The flow diagram of search strategy and study selection for this meta-analysis is presented in Supplementary Figure S1.

\section{Study characteristics}

Table 1A, 1B and $1 \mathrm{C}$ jointly show the baseline characteristics of 9 articles that assessed circulating apelin concentration between CAD patients and controls $[13,15,16,18-23]$. As 2 articles provided the results by CAD subtypes $[16,18]$ and 1 article by sex [23], there were 13 studies in pooled analysis, including 1021 CAD patients and 654 controls. Year of publication ranged from 2010 to 2016. Six studies were conducted in Asian countries, 4 studies in European countries and 3 studies in Middle Eastern countries. Six studies reported circulating apelin changes in CAD patients, 4 studies in acute coronary syndrome (ACS) patients and 3 studies in acute myocardial infarction (AMI) patients. Apelin concentration was measured in plasma by 7 studies and in serum by 6 studies. Age and sex were reported to be matched between CAD patients and controls by 6 studies. Total sample size ranged from 7 to 196 in patients, and from 27 to 171 in controls.

Table 2A and 2B jointly show the baseline characteristics of 3 articles that assessed the association of APLNR gene rs9943582 polymorphism with CAD risk [24-26]. As 1 study provided the results by sex [25], 1 study by both sex and blood pressure [26] and 1 study by both ethnicity and CAD subtype [24], there were 10 studies in pooled analysis, including 5975 CAD patients and 4717 controls. Year of publication ranged from 2009 to 2015. All 10 studies were conducted in Asian countries ( 6 in China, 2 in Korea and 2 in Japan). Six studies reported the association of rs9943582 with CAD risk, 2 studies with MI risk and 2 studies with risk of angina pectoris. Likewise, 6 studies had rs9943582 genotyped by PCR-related methods and 4 studies by TaqMan technique. Age and sex was reported to be matched between patients and controls by only 2 studies. Total sample size ranged from 46 to 1056 in patients, and from 349 to 1275 in controls.

Supplementary Table S1 shows the baseline characteristics of 3 articles that assessed the changes of circulating apelin after surgical treatment for CAD [17, $27,28]$. As 1 article provided the results by the operation, there were 4 studies, involving 202 patients in pooled analysis. Two studies adopted primary percutaneous coronary intervention, 1 study adopted off-pump coronary artery bypass surgery and 1 study adopted onpump coronary artery bypass surgery. Circulating apelin concentration was measured before the surgery and at the $5^{\text {th }}$ day after the surgery for CAD.

\section{Overall estimates}

In overall analysis, circulating apelin concentration was significantly lower in CAD patients than in controls (WMD: $-1.285 \mathrm{ng} / \mathrm{mL}, 95 \% \mathrm{CI}:-1.790$ to $-0.780, P<$ 0001), while statistical heterogeneity was significant ( $\left.I^{2}: 99.3 \%, P<0.001\right)$ (Figure 1: the UPPER panel). As two studies involved controls with atrial fibrillation [13] and stable angina [21], respectively, a sensitivity analysis by excluding the two studies found the reduction in circulating apelin concentration was still significant (WMD: $-1.465 \mathrm{ng} / \mathrm{mL}, 95 \% \mathrm{CI}:-2.024$ to $-0.906, P<$ $0001)$, with significant heterogeneity $(I * 99.3 \%, P<$ $0.001)$. The cumulative and influential analyses were displayed in Supplementary Figure S2. The likelihood of publication bias was low as illustrated by both Begg's funnel plot and filled funnel plot (Figure 2A and 2B), as well as by both Begg's test $(P=0.428)$ and Egger's test $(P$ $=0.187)$. In addition, differences of other clinical markers between CAD patients and controls were also tested, as shown in Table 3. Circulating FBG (WMD: $27.471 \mathrm{mg} /$ dL, 95\% CI: 6.350 to 48.591 ), fasting insulin (WMD: $5.748 \mathrm{mU} / \mathrm{L}, 95 \% \mathrm{CI}: 2.682$ to 8.814 ), TG (WMD: 11.783 $\mathrm{mg} / \mathrm{dL}, 95 \% \mathrm{CI}: 0.113$ to 23.453 ) and HOMA-IR (WMD: $2.360,95 \% \mathrm{CI}: 0.222$ to 1.498 ) levels were significantly higher in CAD patients than in controls, while circulating HDLC (WMD: $-4.020 \mathrm{mg} / \mathrm{dL}, 95 \% \mathrm{CI}:-5.801$ to -2.239 ) was significantly lower. Statistical heterogeneity was nonsignificant for the comparisons of circulating $\mathrm{TG}$, HDLC and $\operatorname{Cr}\left(I^{2}: 18.5 \%, 3.2 \%\right.$ and $0.0 \%$, respectively). Also, there was no indication of publication bias for all 

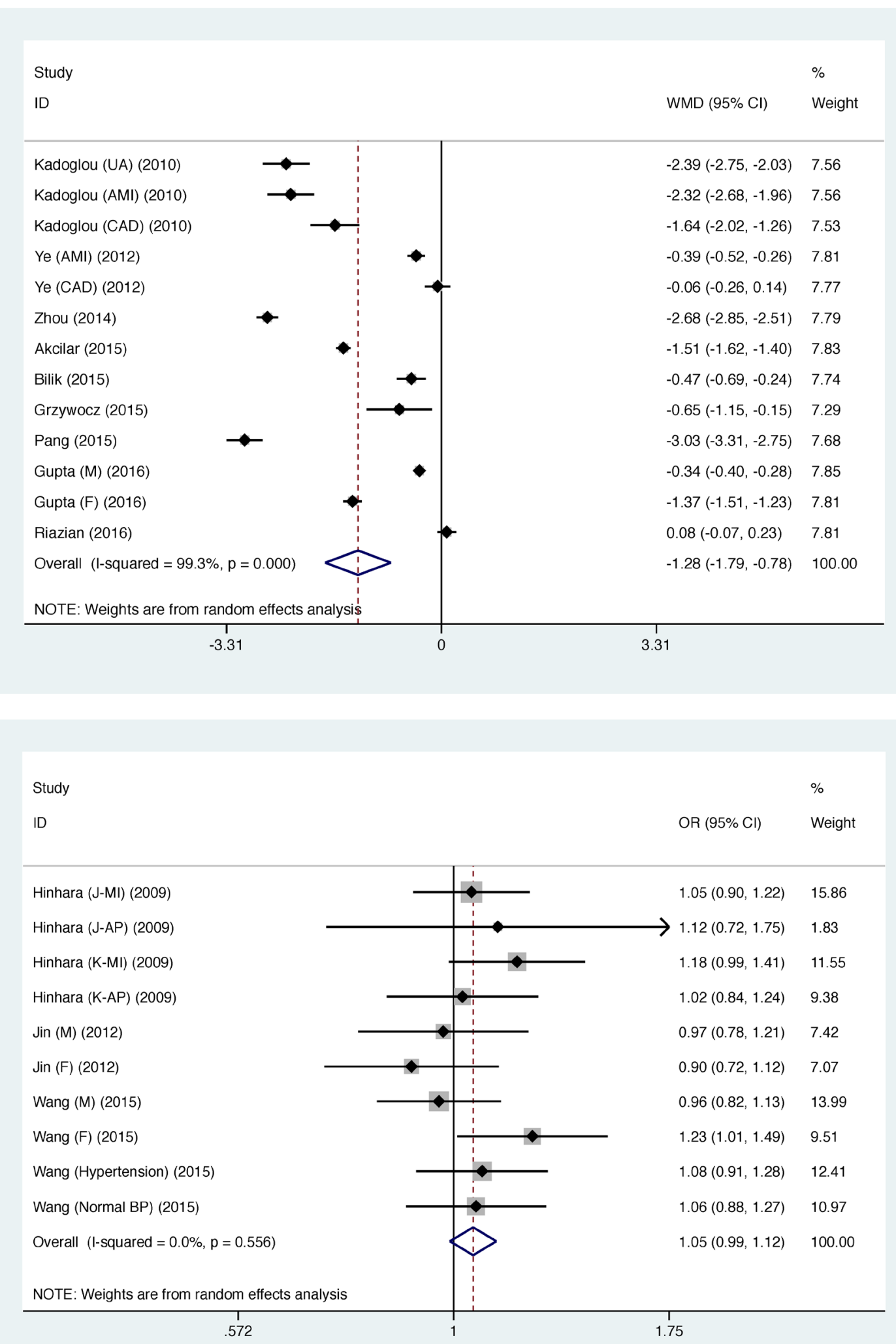

Figure 1: Overall weighted mean difference in circulating apelin concentration between patients with coronary artery disease (CAD) and controls (Upper panel), and overall prediction of APLNR gene rs9943582 polymorphism for CAD risk under the allelic model (Lower panel). Abbreviations: WMD, weighted mean difference; OR, odds ratio; $95 \%$ CI, 95\% confidence interval. 
Table 1A: The baseline characteristics of eligible studies for circulating apelin changes between CAD patients and controls

\begin{tabular}{|c|c|c|c|c|c|c|c|c|c|}
\hline \multirow{2}{*}{ First author } & \multirow{2}{*}{ Year } & \multirow{2}{*}{ Country } & \multirow{2}{*}{ Endpoint } & \multirow{2}{*}{ Diagnosis } & \multirow{2}{*}{ Match } & \multirow{2}{*}{ Sample size } & \multicolumn{3}{|c|}{ Apelin } \\
\hline & & & & & & & Source & ELISA kit Co. & C. $(\mathrm{ng} / \mathrm{mL})$ \\
\hline Kadoglou (UA) & 2010 & Greece & ACS & Electrocardiogram & age, sex & $80 / 72$ & Serum & $\mathrm{NA}$ & $0.60 / 2.99$ \\
\hline Kadoglou (AMI) & 2010 & Greece & AMI & Electrocardiogram & age, sex & $115 / 72$ & Serum & NA & $0.67 / 2.99$ \\
\hline Kadoglou (CAD) & 2010 & Greece & CAD & Angiography & age, sex & $88 / 72$ & Serum & NA & $1.35 / 2.99$ \\
\hline Ye (AMI) & 2012 & China & AMI & Angiography & NA & $87 / 39$ & Plasma & Phoenix Pharmaceuticals & $2.21 / 2.60$ \\
\hline $\mathrm{Ye}(\mathrm{CAD})$ & 2012 & China & CAD & Angiography & NA & $33 / 39$ & Plasma & Phoenix Pharmaceuticals & $2.54 / 2.60$ \\
\hline Zhou & 2014 & China & ACS & Arteriography & age, sex & $196 / 171$ & Plasma & Phoenix Pharmaceuticals & $0.54 / 3.22$ \\
\hline Akcilar & 2015 & Turkey & CAD & Angiography & age, sex & $158 / 118$ & Plasma & Cusabio Biotech & $6.13 / 7.64$ \\
\hline Bilik & 2015 & Turkey & CAD & Angiography & NA & $26 / 28$ & Serum & Phoenix Pharmaceuticals & $0.18 / 0.65$ \\
\hline Grzywocz & 2015 & Poland & AMI & Echocardiography & age, sex & $104 / 27$ & Serum & NA & $0.99 / 1.64$ \\
\hline Pang & 2015 & China & CAD & Arteriography & NA & $35 / 40$ & Plasma & Phoenix Pharmaceuticals & $1.37 / 4.40$ \\
\hline Gupta (M) & 2016 & India & ACS & Electrocardiogram & $\mathrm{NA}$ & $53 / 60$ & Plasma & USCN Life Science & $1.18 / 1.52$ \\
\hline Gupta (F) & 2016 & India & ACS & Electrocardiogram & NA & $7 / 58$ & Plasma & USCN Life Science & $0.79 / 2.16$ \\
\hline Riazian & 2016 & Iran & CAD & Angiography & NA & $39 / 41$ & Serum & Cusabio Biotech & $0.37 / 0.29$ \\
\hline
\end{tabular}

Table 1B: The baseline characteristics of eligible studies for circulating apelin changes between CAD patients and controls

\begin{tabular}{|c|c|c|c|c|c|c|c|}
\hline First author & Age (yrs) & Males & BMI $\left(\mathrm{kg} / \mathrm{m}^{2}\right)$ & Hypertension & $\begin{array}{l}\text { Diabetes } \\
\text { mellitus }\end{array}$ & Family history of CAD & Smoking \\
\hline Kadoglou (UA) & $65.5 / 60.1$ & $0.675 / 0.653$ & $28.3 / 27.7$ & $0.475 / 0.000$ & $0.250 / 0.000$ & $0.000 / 0.000$ & $0.675 / 0.222$ \\
\hline Kadoglou (AMI) & $63.2 / 60.1$ & $0.774 / 0.653$ & $28.9 / 27.7$ & $0.487 / 0.000$ & $0.339 / 0.000$ & $0.000 / 0.000$ & $0.617 / 0.222$ \\
\hline Kadoglou (CAD) & $68.4 / 60.1$ & $0.773 / 0.653$ & $30.4 / 27.7$ & $0.523 / 0.000$ & $0.261 / 0.000$ & $0.000 / 0.000$ & $0.295 / 0.222$ \\
\hline Ye (AMI) & $69.0 / 66.0$ & $0.770 / 0.769$ & $24.1 / 24.5$ & $0.805 / 0.846$ & $0.000 / 0.000$ & $\mathrm{NA} / 0.000$ & $0.000 / 0.000$ \\
\hline Ye (CAD) & $64.0 / 66.0$ & $0.727 / 0.769$ & $24.3 / 24.5$ & $0.879 / 0.846$ & $0.000 / 0.000$ & $\mathrm{NA} / 0.000$ & $0.000 / 0.000$ \\
\hline Zhou & $55.7 / 56.9$ & $0.631 / 0.628$ & NA & $0.368 / 0.000$ & NA & $0.072 / 0.000$ & $0.523 / 0.514$ \\
\hline Akcilar & $64.2 / 61.5$ & $0.741 / 0.542$ & NA & NA & NA & NA & NA \\
\hline Bilik & $53.6 / 51.6$ & $0.731 / 0.643$ & $28.1 / 26.7$ & $0.577 / 0.428$ & $0.154 / 0.214$ & NA & $0.500 / 0.428$ \\
\hline Grzywocz & $62.0 / 63.0$ & $0.740 / 0.296$ & $27.1 / 28.5$ & $0.78 / 0.93$ & NA & $0.000 / 0.000$ & $0.440 / 0.330$ \\
\hline Pang & $68.1 / 67.4$ & $0.486 / 0.475$ & NA & $1.000 / 0.000$ & $0.000 / 0.000$ & NA & $0.000 / 0.000$ \\
\hline Gupta (M) & $46.9 / 47.1$ & $1.000 / 1.000$ & $24.1 / 22.1$ & NA/ 0.000 & $\mathrm{NA} / 0.000$ & $0.000 / 0.000$ & $0.585 / 0.450$ \\
\hline Gupta (F) & $54.1 / 39.9$ & $0.000 / 0.000$ & $26.0 / 24.7$ & $\mathrm{NA} / 0.000$ & $\mathrm{NA} / 0.000$ & $0.000 / 0.000$ & $0.143 / 0.017$ \\
\hline Riazian & $66.6 / 70.0$ & $0.564 / 0.415$ & $30.5 / 29.7$ & $0.846 / 0.902$ & $0.359 / 0.366$ & $0.000 / 0.000$ & NA \\
\hline
\end{tabular}

other clinical markers in viewing both Begg's test and Egger's test (Table 3).

Based on all eligible articles, four polymorphisms, two in apelin gene (rs3761581 and rs56204867) and two in APLNR gene (rs948847 and rs9943582) were summarized in association with CAD risk. Considering the small number of studies for polymorphisms rs3761581 $(N=3), \operatorname{rs} 56204867(N=3)$ and $\operatorname{rs} 948847(N=2)$, with the null association with CAD risk, only genotype distributions of rs9943582 $(N=10)$ were presented and compared between CAD patients and controls (Table 2B). No studies reported significant deviations from the HardyWeinberg equilibrium for rs 9943582 polymorphism in control groups at a significance level of 5\%.

For the association of APLNR gene rs9943582 polymorphism with CAD risk, the mutant $\mathrm{T}$ allele was associated with a $5.2 \%$ increased risk relative to the wild C allele (OR: $1.052,95 \%$ CI: 0.990 to $1.117, P=$ $0.100)$ (Figure 1: the LOWER panel), and there was no observable heterogeneity $\left(I^{2}: 0.0 \%, P=0.556\right)$ and a low probability of publication bias (Figure $2 \mathrm{C}$ and 2D) ( $P$ for Begg's test: 0.721 and $P$ for Egger's test: 0.953). Circulating apelin concentration was increased after coronary operation (WMD: $2.011 \mathrm{ng} / \mathrm{mL}, 95 \%$ CI: 0.541 to $3.481, P=0.007)$, but with strong evidence of heterogeneity $\left(I^{2}: 98.0 \%, P<0.001\right)$ (data not shown).

\section{Stratified estimates}

In attempting to seek possible explanations of significant heterogeneity, stratifying studies that assessed 
Table 1C: The baseline characteristics of eligible studies for circulating apelin changes between CAD patients and controls

\begin{tabular}{lcccccccc}
\hline First author & FBG $(\mathbf{m g} / \mathbf{d L})$ & $\begin{array}{c}\text { Fasting insulin } \\
(\mathbf{m U} / \mathbf{L})\end{array}$ & TG $(\mathbf{m g} / \mathbf{d L})$ & $\mathbf{T C}(\mathbf{m g} / \mathbf{d L})$ & HDLC $(\mathbf{m g} / \mathbf{d L})$ & LDLC $(\mathbf{m g} / \mathbf{d L})$ & HOMA-IR & Cr $(\boldsymbol{\mu} \mathbf{m o l} / \mathbf{L})$ \\
\hline Kadoglou (UA) & $153.5 / 97.3$ & $11.85 / 5.78$ & $149.2 / 140.2$ & $189.8 / 206.5$ & $42.5 / 47.4$ & $122.6 / 131.1$ & $4.49 / 1.39$ & NA \\
Kadoglou (AMI) & $164.3 / 97.3$ & $12.42 / 5.78$ & $141.3 / 140.2$ & $189.8 / 206.5$ & $42.8 / 47.4$ & $119.6 / 131.1$ & $5.04 / 1.39$ & NA \\
Kadoglou (CAD) & $134.7 / 97.3$ & $7.82 / 5.78$ & $147.5 / 140.2$ & $179.9 / 206.5$ & $45.4 / 47.4$ & $109.3 / 131.1$ & $1.49 / 1.39$ & NA \\
Ye (AMI) & NA & NA & $160.2 / 134.5$ & NA & NA & NA & NA & NA \\
Ye (CAD) & NA & NA & $173.5 / 134.5$ & NA & NA & NA & NA & NA \\
Zhou & NA & NA & NA & NA & NA & NA & NA & $99.5 / 98.7$ \\
Akcilar & NA & NA & NA & NA & NA & NA & NA & NA \\
Bilik & $106.4 / 124.6$ & NA & $145.7 / 184.8$ & $196.1 / 195.4$ & $41.2 / 40.9$ & $128.7 / 113.4$ & NA & $70.7 / 70.7$ \\
Grzywocz & $111.0 / 93.0$ & NA & $148.0 / 119.0$ & $193.0 / 173.0$ & $44.0 / 50.0$ & $124.0 / 99.0$ & NA & $84.9 / 84.0$ \\
Pang & NA & NA & NA & NA & NA & NA & NA & NA \\
Gupta (M) & NA & NA & NA & NA & NA & NA & NA & NA \\
Gupta (F) & NA & NA & NA & NA & NA & NA & NA & NA \\
Riazian & $119.7 / 116.6$ & $18.84 / 8.94$ & $159.2 / 147.1$ & $171.5 / 158.4$ & $37.1 / 43.1$ & $104.4 / 85.7$ & $5.60 / 2.90$ & NA \\
\hline
\end{tabular}

Abbreviations: UA, unstable angina; AMI, acute myocardial infarction; CAD, coronary artery disease; ACS, acute coronary syndrome; M, males; F, females; NA, not available; C., apelin concentration; BMI, body mass index; FBG, fasting blood glucose; TG, triglyceride; TC, total cholesterol; HDLC, high-density lipoprotein cholesterol; LDLC, low-density lipoprotein cholesterol; HOMA-IR, homeostatic model assessment for insulin resistance; Cr, creatinine. Apelin, age, males, BMI, hypertension, diabetes mellitus, family history of CAD, smoking, FBG, insulin, TG, TC, HDLC, LDLC, HOMA-IR and Cr were expressed as mean or per cent in patients/controls.

Table 2A: The baseline characteristics of eligible studies for $A P L N R$ gene rs 9943582 polymorphism in association with CAD risk

\begin{tabular}{|c|c|c|c|c|c|c|c|c|c|c|c|}
\hline First author & Year & Country & Endpoint & Genotyping & Match & Sample size & Smoking & Age (yrs) & Males & SBP (mmHg) & DBP (mmHg) \\
\hline Hinhara (J-MI) & 2009 & Japan & MI & TaqMan & NA & $575 / 1275$ & $0.739 / \mathrm{NA}$ & $59.3 / 39.0$ & $0.836 / 0.559$ & NA & NA \\
\hline Hinhara (J-AP) & 2009 & Japan & $\mathrm{AP}$ & TaqMan & NA & $46 / 1275$ & NA & $65.3 / 39.0$ & $0.755 / 0.559$ & NA & NA \\
\hline Hinhara (K-MI) & 2009 & Korea & MI & TaqMan & NA & $490 / 693$ & $0.714 / \mathrm{NA}$ & $61.8 / 57.7$ & $0.816 / 0.644$ & NA & NA \\
\hline Hinhara (K-AP) & 2009 & Korea & AP & TaqMan & NA & $368 / 693$ & $0.593 / \mathrm{NA}$ & $63.1 / 57.7$ & $0.698 / 0.644$ & NA & NA \\
\hline $\operatorname{Jin}(\mathrm{M})$ & 2012 & China & $\mathrm{CAD}$ & PCR-LDR & age, sex & $533 / 359$ & NA & $60.7 / 63.0$ & $1.000 / 1.000$ & $135.2 / 137.0$ & $81.2 / 82.5$ \\
\hline $\operatorname{Jin}(F)$ & 2012 & China & $\mathrm{CAD}$ & PCR-LDR & age, sex & $461 / 349$ & NA & $66.0 / 65.1$ & $0.000 / 0.000$ & $139.5 / 137.4$ & $80.4 / 82.6$ \\
\hline Wang (M) & 2015 & China & CAD & PCR-HRM & NA & $1056 / 525$ & & & $1.000 / 1.000$ & & \\
\hline Wang (F) & 2015 & China & CAD & PCR-HRM & NA & $695 / 414$ & & & $0.000 / 0.000$ & & \\
\hline Wang (High BP) & 2015 & China & CAD & PCR-HRM & NA & $1030 / 525$ & $0.338 / 0.256$ & $61.6 / 63.7$ & & $136.0 / 126.0$ & $92.0 / 89.0$ \\
\hline Wang (Normal BP) & 2015 & China & $\mathrm{CAD}$ & PCR-HRM & NA & $721 / 497$ & & & & & \\
\hline
\end{tabular}

circulating apelin concentration between CAD patients and controls by continent, endpoint, apelin source, matching status, sample size, obesity and hypertension were conducted, respectively (Table 4). By continent, circulating apelin concentration was significantly reduced in patients relative to controls from European (WMD: $-1.767 \mathrm{ng} / \mathrm{mL}, P<0.001$ ) and Asian (WMD: $-1.307 \mathrm{ng} /$ $\mathrm{mL}, P=0.002)$ countries, while no significance was detected in populations from Middle Eastern countries. By clinical endpoint, significant reduction in circulating apelin concentration was found in ACS (WMD: -1.690 $\mathrm{ng} / \mathrm{mL}, P=0.005$ ) or CAD (WMD: $-1.099 \mathrm{ng} / \mathrm{mL}, P$ $=0.011)$ patients when compared with controls, and only marginally significant reduction was found in AMI patients. By apelin source, circulating apelin concentration was reduced significantly when measured in serum (WMD: $-1.226 \mathrm{ng} / \mathrm{mL}, P=0.007$ ) and in plasma (WMD: $-1.336 \mathrm{ng} / \mathrm{mL}, P<0.001$ ). By matching status for age and sex, the reduction magnitude in circulating apelin concentration was stronger in studies with matched patients and controls (WMD: $-1.881 \mathrm{ng} / \mathrm{mL}, P<0.001$ ) than those without matching reports. Finally, median cutoff value based on total sample size of all eligible studies was 125 , and circulating apelin concentration was reduced with greater magnitude for studies with total sample size $\geq 125$ (WMD: $-1.657 \mathrm{ng} / \mathrm{mL}, P<0.001$ ) than those with total sample size $<125$ (WMD: $-0.857, P=0.007$ ). By obesity status in controls, the reduction in circulating apelin concentration was comparable between studies with normal weight controls (BMI $<25 \mathrm{~kg} / \mathrm{m}^{2}$ ) (WMD: -1.336 $\mathrm{ng} / \mathrm{mL}, P<0.001)$ and obese controls $\left(\mathrm{BMI} \geq 25 \mathrm{~kg} / \mathrm{m}^{2}\right)$ (WMD: $-1.226 \mathrm{ng} / \mathrm{mL}, P=0.007$ ). By hypertension status 
Table 2B: The baseline characteristics of eligible studies for $A P L N R$ gene rs9943582 polymorphism in association with CAD risk

\begin{tabular}{|c|c|c|c|c|c|c|c|c|c|}
\hline First author & BMI $\left(\mathrm{kg} / \mathrm{m}^{2}\right)$ & TG (mg/dL) & TC (mg/dL) & $\begin{array}{l}\text { HDLC } \\
(\mathrm{mg} / \mathrm{dL})\end{array}$ & $\begin{array}{l}\text { LDLC } \\
(\mathrm{mg} / \mathrm{dL})\end{array}$ & FBG (mg/dL) & $\mathrm{Cr}(\mu \mathrm{mol} / \mathrm{L})$ & P: CC/CT/TT & C: CC/CT/TT \\
\hline Hinhara (J-MI) & $23.6 / \mathrm{NA}$ & NA & NA & NA & NA & NA & NA & $271 / 250 / 54$ & $636 / 505 / 131$ \\
\hline Hinhara (J-AP) & NA & NA & $\mathrm{NA}$ & NA & NA & $\mathrm{NA}$ & NA & $20 / 22 / 4$ & $636 / 505 / 131$ \\
\hline Hinhara (K-MI) & $24.6 / \mathrm{NA}$ & NA & NA & NA & $\mathrm{NA}$ & NA & NA & $228 / 204 / 58$ & $351 / 280 / 62$ \\
\hline Hinhara (K-AP) & $25.1 / \mathrm{NA}$ & NA & NA & NA & NA & NA & NA & $180 / 158 / 30$ & $351 / 280 / 62$ \\
\hline $\operatorname{Jin}(\mathrm{M})$ & $25.7 / 25.3$ & $162.0 / 157.5$ & $169.0 / 169.1$ & $39.5 / 44.9$ & $102.9 / 99.5$ & $110.7 / 94.3$ & $97.6 / 90.4$ & $312 / 186 / 35$ & $207 / 128 / 24$ \\
\hline Jin $(F)$ & $25.0 / 24.9$ & $175.2 / 155.8$ & $186.8 / 186.9$ & $47.6 / 51.9$ & $110.3 / 110.3$ & $110.5 / 97.4$ & $75.5 / 72.5$ & $273 / 152 / 36$ & $195 / 124 / 30$ \\
\hline Wang (M) & NA & & & & & & & $594 / 382 / 80$ & $336 / 223 / 49$ \\
\hline Wang (F) & NA & $1600 / 1602$ & $170-5 / 1640$ & $426 / 464$ & $1045 / 037$ & NA & NA & $356 / 271 / 68$ & $230 / 159 / 25$ \\
\hline Wang (High BP) & NA & & & & & & & $577 / 361 / 92$ & $302 / 183 / 40$ \\
\hline Wang (Normal BP) & NA & & & & & & & $373 / 292 / 56$ & $264 / 199 / 34$ \\
\hline
\end{tabular}

Abbreviations: J-MI, myocardial infarction in Japanese; J-AP, angina pectoris in Japanese; K-MI, myocardial infarction in Koreans; K-AP, angina pectoris in Koreans; M, males; F, females; BP, blood pressure; CAD, coronary artery disease; SBP, systolic blood pressure; DBP, diastolic blood pressure; BMI, body mass index; TG, triglyceride; TC, total cholesterol; HDLC, high-density lipoprotein cholesterol; LDLC, low-density lipoprotein cholesterol; FBG, fasting blood glucose; Cr, creatinine; NA, not available.

(A)

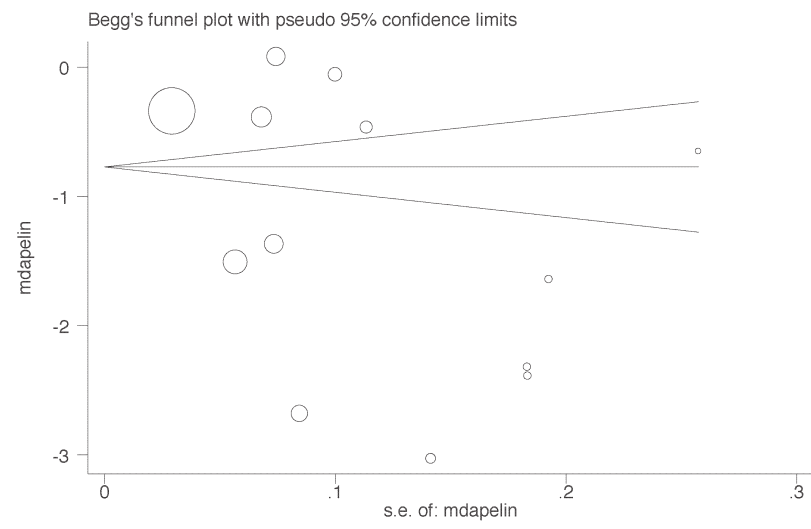

(C)

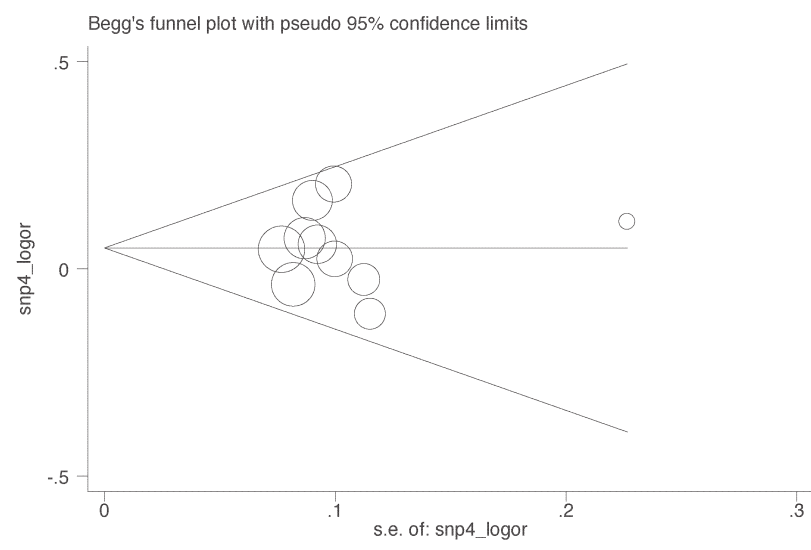

(B)

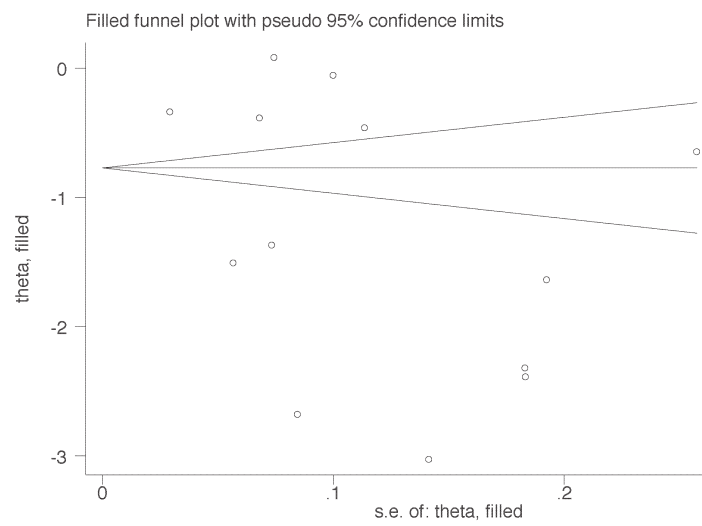

(D)

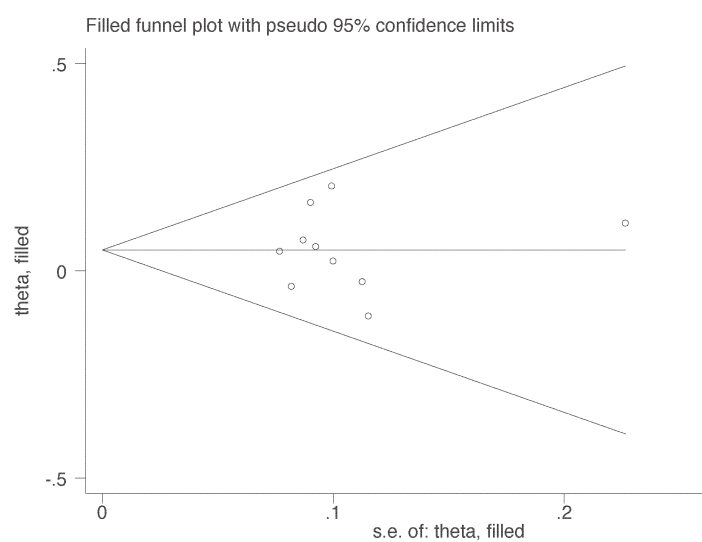

Figure 2: Begg's and filled funnel plots for circulating apelin difference between patients with coronary artery disease (CAD) and controls (A and B) and the prediction of APLNR gene rs9943582 polymorphism for CAD risk under the allelic model (C and D). 
Table 3: Overall effect-size estimates of other clinical markers and the tests for between-study heterogeneity and publication bias

\begin{tabular}{lcccccccc}
\hline Clinical markers & Studies (N) & WMD & $\mathbf{9 5 \%} \mathbf{C I}$ & $\boldsymbol{P}$ & $\boldsymbol{I}^{\mathbf{2}}$ & $\boldsymbol{P}$ & $\boldsymbol{P}_{\text {Begg's test }}$ & $\boldsymbol{P}_{\text {Egger's test }}$ \\
\hline FBG & 6 & 27.471 & 6.350 to 48.591 & 0.011 & $92.7 \%$ & $<0.001$ & 1.000 \\
Fasting insulin & 4 & 5.748 & 2.682 to 8.814 & $<0.001$ & $98.0 \%$ & $<0.001$ & 1.000 & 0.640 \\
TG & 8 & 11.783 & 0.113 to 23.453 & 0.048 & $18.5 \%$ & 0.283 & 0.536 & 0.320 \\
TC & 6 & -5.121 & -20.250 to 10.009 & 0.507 & $81.2 \%$ & $<0.001$ & 0.260 & 0.192 \\
HDLC & 6 & -4.020 & -5.801 to -2.239 & $<0.001$ & $3.2 \%$ & 0.396 & 0.707 \\
LDLC & 6 & 2.238 & -13.434 to 17.911 & 0.780 & $86.1 \%$ & $<0.001$ & 0.133 \\
HOMA-IR & 4 & 2.360 & 0.222 to 1.498 & 0.031 & $97.9 \%$ & $<0.001$ & 0.149 \\
Cr & 3 & 0.670 & -2.574 to 3.914 & 0.686 & $0.0 \%$ & 0.981 & NA
\end{tabular}

Abbreviations: WMD, weighted mean difference; 95\% CI, 95\% confidence interval; FBG, fasting blood glucose; TG, triglyceride; TC, total cholesterol; HDLC, high-density lipoprotein cholesterol; LDLC, low-density lipoprotein cholesterol; HOMA-IR, homeostatic model assessment for insulin resistance; $\mathrm{Cr}$, creatinine; NA, not available.

Table 4: Weighted mean difference in circulating apelin concentration between CAD patients and controls in stratified analyses

\begin{tabular}{|c|c|c|c|c|c|c|}
\hline Characteristics & Subgroups & Studies (N) & $\begin{array}{c}\text { Sample } \\
\text { size }^{*}\end{array}$ & WMD & $95 \% \mathrm{CI}$ & $P$ \\
\hline \multirow[t]{3}{*}{ Continent } & Europe & 4 & $387 / 243$ & -1.767 & -2.466 to -1.069 & $<0.001$ \\
\hline & Asia & 6 & $411 / 407$ & -1.307 & -2.128 to -0.487 & 0.002 \\
\hline & Middle East & 3 & $223 / 187$ & -0.633 & -1.716 to 0.450 & 0.252 \\
\hline \multirow[t]{3}{*}{ Clinical endpoint } & ACS & 4 & $336 / 361$ & -1.690 & -2.880 to -0.501 & 0.005 \\
\hline & AMI & 3 & $306 / 138$ & -1.118 & -2.390 to 0.150 & 0.085 \\
\hline & $\mathrm{CAD}$ & 6 & $379 / 338$ & -1.099 & -1.950 to -0.248 & 0.011 \\
\hline \multirow[t]{2}{*}{ Apelin source } & Serum & 6 & $452 / 312$ & -1.226 & -2.112 to -0.340 & 0.007 \\
\hline & Plasma & 7 & $569 / 525$ & -1.336 & -2.040 to -0.632 & $<0.001$ \\
\hline \multirow[t]{2}{*}{ Matched status } & age, sex & 6 & $741 / 532$ & -1.881 & -2.457 to -1.304 & $<0.001$ \\
\hline & NA & 7 & $280 / 305$ & -0.788 & -1.296 to -0.279 & 0.002 \\
\hline \multirow[t]{2}{*}{ Sample size } & $\geq 125$ & 7 & $828 / 571$ & -1.657 & -2.378 to -0.936 & $<0.001$ \\
\hline & $<125$ & 6 & $193 / 266$ & -0.857 & -1.482 to -0.232 & 0.007 \\
\hline \multirow[t]{2}{*}{ Obesity** } & $\mathrm{BMI}<25 \mathrm{~kg} / \mathrm{m}^{2}$ & 4 & $180 / 196$ & -1.336 & -2.040 to 0.632 & $<0.001$ \\
\hline & $\mathrm{BMI} \geq 25 \mathrm{~kg} / \mathrm{m}^{2}$ & 6 & $452 / 312$ & -1.226 & -2.112 to -0.340 & 0.007 \\
\hline \multirow[t]{2}{*}{ Hypertension** } & $\begin{array}{l}\text { Normotensive } \\
\text { controls }\end{array}$ & 7 & $574 / 545$ & -1.964 & -2.904 to -1.023 & $<0.001$ \\
\hline & Others & 5 & $289 / 174$ & -1.266 & -1.813 to -0.719 & 0.042 \\
\hline
\end{tabular}

Abbreviations: ACS, acute coronary syndrome; AMI, acute myocardial infarction; CAD, coronary artery disease; BMI, body mass index; WMD, weighted mean difference; 95\% CI, 95\% confidence interval; NA, not available. *Sample size was expressed in CAD patients/controls. **Obesity and hypertension were determined in control groups.

in controls, the reduction was even stronger in the studies involving normotensive controls (WMD: $-1.964 \mathrm{ng} / \mathrm{mL}$, $P<0.001$ ) than the other studies (WMD: $-1.266 \mathrm{ng} / \mathrm{mL}$, $P=0.042)$.

\section{DISCUSSION}

This meta-analysis summarized the results from 15 published articles with the purpose of assessing circulating apelin concentration between CAD patients and controls, the prediction of APLNR gene rs9943582 polymorphism for CAD risk and circulating apelin changes after surgical treatment for CAD. Our metaanalytical results collectively suggested that circulating apelin was a prominent athero-protective marker against the development of CAD. As far as we know, this is so far the first meta-analysis that systematically explores the association of apelin and APLNR, either circulating concentration change or genetic variation, with $\mathrm{CAD}$ risk 
by reviewing the current literature.

Previous studies have suggested that circulating apelin was correlated directly with obesity, and in a broader sense, with obesity-related cardiovascular events [29-31]. Apelin when binding to APLNR may regulate cardiovascular system through several mechanisms. A key mechanism with multiple consequences involves the stimulation of heart rate, the contractility and propagation of action potential, the formation of atherosclerotic plaques, the nitric oxide-mediated vasodilatation and the vascular tone regulation via inhibiting the electrical activity of vasopressin-releasing neurons [13, 32-34]. The results of the present meta-analysis based on 13 independent studies confirmed the findings of the most previous studies by convincingly demonstrating that circulating apelin concentration was significantly lower in CAD patients than in controls. In spite of unaccountable heterogeneity between studies, our results were less likely to be confounded by the presence of publication bias, as illustrated by both funnel plots and associated tests. In particular, the magnitude of circulation apelin reduction was remarkably potentiated in the studies with age- and sex-matched patients and controls, as well as in the studies with total sample size $\geq 125$, which added to the credibility of our overall observations. Moreover, in view of divergent tendencies for circulating apelin concentration across continental populations, it can be speculated that the regulatory role of apelin in cardiovascular system, if potential involvement, might be contingent on lifestyle or environmental exposure [35, 36]. Unfortunately, the possible interactions between circulating apelin and lifestyles or environmental factors were not systematically assessed in all eligible studies, and thus it is beyond the scope of our work. It is hence expected that future efforts should focus in greater details on these promising interactions.

Besides circulating apelin difference, we also examined the susceptibility of apelin and APLNR genetic polymorphism(s) to CAD risk. Considering the statistical power, only a promoter polymorphism in APLNR gene, rs9943582, was summarized and presented. The mutant allele of this polymorphism was found to confer a marginally increased risk for CAD in overall analysis that was not subject to the disturbance of between-study heterogeneity and publication bias. It is universally believed that CAD is a multifactorial polygenic disease $[37,38]$, and the increased risk conferred by a single allele in APLNR gene is extremely small [25]. Although current knowledge upon the genetic underpinnings of CAD is still far from being complete, it is essential to narrow down genetic variants that could be translated to routine clinical practice. We agree that the fine-mapping of apelin and APLNR genetic defects is encouraged to aid in unveiling the pathogenesis of CAD.

Evidence from circulating apelin changes between CAD patients and controls cannot determine causality, as the changes could be secondary to CAD process itself rather than declaring a causal role. To shed some light on this issue, we additionally summarized the changes of circulating apelin concentration after surgical treatment for $\mathrm{CAD}$, and our results on the basis of 4 studies revealed that circulating apelin concentration was increased remarkably at the $5^{\text {th }}$ day of post-surgery relative to that before the surgery, which can, at least in part, support a causal role of circulating apelin in the aetiology of CAD. Nevertheless, because our results are based on only a small number of studies, it is undoubted that larger studies should be performed to confirm the causal relevance between circulating apelin and CAD in the future.

\section{Study limitations}

Several limitations of the present study should be acknowledged. As always, selection bias inevitably arises from a meta-analysis, especially when search scope is restricted to the English-language journals only [39]. However, as illustrated by both Begg's and filled funnel plots, as well as both Begg's and Egger's tests, the likelihood of publication bias was low for overall comparisons. In addition, as with the majority of meta-analyses, we in this study did not have access to individual level data, and we were not able to adjust for some established confounding factors (such as obesity) and assess the possible gene-environment interactions. Moreover, although this is the first meta-analysis on the association of apelin and APLNR with CAD, some subgroup analyses carried insufficient statistical power, which limited the extrapolation of our findings. Furthermore, only circulating apelin concentration was analysed, and it will be of added interest to see its changes in coronary artery tissue. It is generally believed that metaanalysis should aim at more than simply derive an overall estimate, as dissection of between-study heterogeneity can yield some valuable information. However, in the present study, performing a wide range of stratified analyses did very little to improve the impact of between-study heterogeneity on overall comparisons. There is generally a need in genetics and epidemiology for much larger and more rigorous studies to help control heterogeneity and lower publication bias.

In conclusion, our meta-analytical results suggested that circulating apelin was a prominent athero-protective marker against the development of CAD. Pending consistent replication and validation, it is anticipated that circulating apelin can be identified as a useful biomarker to allow early detection of individuals prone to the development of CAD, and further it can be proposed as an optional treatment target among high-risk CAD patients. Moreover, further studies are warranted to explore underlying mechanisms. 


\section{MATERIALS AND METHODS}

This meta-analysis was based on observational data and it was conducted following the guidelines in the Meta-analysis Of Observational Studies in Epidemiology (MOOSE) statement [40].

\section{Search strategies}

Online computational search was confined to four literature platforms - MEDLINE, EMBASE, Web of Knowledge and Google Scholar. Search process was independently accomplished by two authors, Tianbao Chen and Bing $\mathrm{Wu}$, using the same MeSH terms acknowledged by all contributing authors. Search results were merged together, with the removal of duplicates by the EndNote X8 on the Macintosh laptop. In addition, the bibliographies of retrieved articles were also searched to make literature coverage as comprehensive as possible.

\section{Article selection}

The full-process of selecting all potential articles was independently accomplished by two authors, Tianbao Chen and Bing $\mathrm{Wu}$, based on two steps. The first step was to remove articles that were not published in the English language, examined apelin in animals or focused on the signalling cascades of apelin-APLNR pathway after reviewing the title and/or abstract. The second step was to access the full text of remaining articles to see whether the results on circulating apelin difference between CAD patients and controls, or the association of apelin and APLNR genetic polymorphism(s) with CAD risk, or circulating apelin changes after surgical treatment for CAD. In the meanwhile, population source of each qualified article was inspected to remove duplicate publications from the same research team. In case of any disagreement, a discussion between the two authors was held until reaching a consensus.

\section{Inclusion and exclusion criteria}

Both inclusion and exclusion criteria applied to each potential article. For inclusion criteria, the clinical endpoint should be CAD and the results were available on circulating apelin concentration or genotype distributions of polymorphism(s) in apelin and APLNR genes between $\mathrm{CAD}$ patients and non-CAD controls, as well as circulating apelin changes after surgical treatment for CAD. Exclusion criteria embraced the publication types in either abstract or poster presentation of international meetings, case report, editorial, letter to the editor, narrative or quantitative reviewer, as well as the investigations on CAD severity or response to drug treatment.

\section{Information collection}

The same two authors, Tianbao Chen and Bing $\mathrm{Wu}$, independently collected the results of interest from each qualified article, including surname of the first author, year of publication, area where study subjects resided in, CAD subtype, diagnostic criteria of both CAD patients and controls, source of controls, apelin assaying method, circulating apelin source, matching status between patients and controls, sample size of each group, circulating apelin concentration between CAD patients and controls or the association of apelin and APLNR genetic polymorphisms with CAD risk or circulating apelin changes after surgical treatment for CAD, as well as the demographic and clinical characteristics such as age, sex, body mass index (BMI), the per cents of hypertension, diabetes mellitus and family CAD history, systolic blood pressure (SBP), diastolic blood pressure (DBP), smoking status and mean levels of circulating triglyceride (TG), total cholesterol (TC), high-density lipoprotein cholesterol (HDLC), low-density lipoprotein cholesterol (LDLC), fasting blood glucose (FBG), fasting insulin, homeostatic model assessment for insulin resistance (HOMA-IR) and creatinine $(\mathrm{Cr})$ in both CAD patients and controls, if appropriate. Information collected by the two authors was computationally compared for accuracy, and any disagreement was checked and solved with consensus.

\section{Statistical analysis}

Mean differences of demographic and clinical characteristics were compared between patients and controls using the unpaired $\mathrm{t}$ test or the Mann-Whitney $\mathrm{U}$ test, as appropriate. Circulating apelin changes between CAD patients and controls or after surgical treatment for CAD were expressed by using weighted mean difference (WMD) and its $95 \%$ confidence interval $(95 \% \mathrm{CI})$, and for the prediction of polymorphism(s) in apelin and APLNR genes for CAD risk by using odds ratio (OR) and its $95 \% \mathrm{CI}$. The effect-size estimates were computed using the random-effects model. Statistical magnitude of between-study heterogeneity was expressed by the $I^{2}$, the inconsistency index, which can be interpreted as the per cent of variability due to heterogeneity between studies rather than a sampling error. A larger $I^{2}$ value indicates a higher probability of heterogeneity, and it is universally accepted that this value over $50 \%$ denotes statistically significant heterogeneity. The likely explanations of heterogeneity were assessed by stratified analyses. Cumulative analysis and influential analysis were also presented. Publication bias, a type of bias referring to the decreased probability of studies' results being published when they are near the null, not statistically significant or otherwise less interesting [41], was graphically illustrated by both Begg's funnel plot and filled funnel plot, and was 
statistically justified by both Begg's test and Egger's linear regression test.

Data management and statistical analyses were accomplished with the aid of packages in the STATA/SE software (version 14.0, StataCorp, Texas, USA) on the Macintosh laptop.

\section{Author contributions}

Tianbao Chen designed the study; Tianbao Chen and Bing $\mathrm{Wu}$ searched the literature, abstracted and checked the data; Tianbao Chen and Rong Lin did statistical analyses; Tianbao Chen and Bing $\mathrm{Wu}$ wrote the manuscript; Tianbao Chen and Rong Lin revised the manuscript.

\section{CONFLICTS OF INTERESTS} interests.

The authors declare that they have no competing

\section{REFERENCES}

1. World Health Organization. Media Centre. The top 10 causes of death. Updated January 2017. Accessed Feb. 2017. http://who.int/mediacentre/factsheets/fs310/en/.

2. World Health Organization. World Health Statistics 2012. Accessed Feb. 2017. http:/www.who.int/gho/publications/ world_health_statistics/EN_WHS2012_Full.pdf.

3. Kavousi M, Desai CS, Ayers C, Blumenthal RS, Budoff MJ, Mahabadi AA, Ikram MA, van der Lugt A, Hofman A, Erbel R, Khera A, Geisel MH, Jockel KH, et al. Prevalence and Prognostic Implications of Coronary Artery Calcification in Low-Risk Women: A Meta-analysis. JAMA. 2016; 316:2126-2134.

4. Hemal K, Pagidipati NJ, Coles A, Dolor RJ, Mark DB, Pellikka PA, Hoffmann U, Litwin SE, Daubert MA, Shah SH, Ariani K, Bullock-Palmer RP, Martinez B, et al. Sex Differences in Demographics, Risk Factors, Presentation, and Noninvasive Testing in Stable Outpatients With Suspected Coronary Artery Disease: Insights From the PROMISE Trial. JACC Cardiovasc Imaging. 2016; 9:337346.

5. Okwuosa TM, Greenland P, Burke GL, Eng J, Cushman M, Michos ED, Ning H, Lloyd-Jones DM. Prediction of coronary artery calcium progression in individuals with low Framingham Risk Score: the Multi-Ethnic Study of Atherosclerosis. JACC Cardiovasc Imaging. 2012; 5:144153.

6. Zheng YY, Xie X, Ma YT, Fu ZY, Ma X, Yang YN, Li XM, Pan S, Adi D, Chen BD, Liu F. Association of C5L2 genetic polymorphisms with coronary artery disease in a Han population in Xinjiang, China. Oncotarget. 2017; 8:8590-96. https://doi.org/10.18632/oncotarget.14305.
7. Sun T, Hu J, Yin Z, Xu Z, Zhang L, Fan L, Zhuo Y, Wang C. Low serum paraoxonase1 activity levels predict coronary artery disease severity. Oncotarget. 2017; 8:19443-19454. https://doi.org/10.18632/oncotarget.14305.

8. Okuma Y, Suda K, Nakaoka H, Katsube Y, Mitani Y, Yoshikane Y, Ichida F, Matsushita T, Shichino H, Shiraishi I, Abe J, Hiroe M, Yoshida T, et al. Serum Tenascin-C as a Novel Predictor for Risk of Coronary Artery Lesion and Resistance to Intravenous Immunoglobulin in Kawasaki Disease- A Multicenter Retrospective Study. Circ J. 2016; 80:2376-2381

9. Shen GQ, Girelli D, Li L, Rao S, Archacki S, Olivieri O, Martinelli N, Park JE, Chen Q, Topol EJ, Wang QK. A novel molecular diagnostic marker for familial and earlyonset coronary artery disease and myocardial infarction in the LRP8 gene. Circ Cardiovasc Genet. 2014; 7:514-520.

10. Berta J, Hoda MA, Laszlo V, Rozsas A, Garay T, Torok S, Grusch M, Berger W, Paku S, Renyi-Vamos F, Masri B, Tovari J, Groger M, et al. Apelin promotes lymphangiogenesis and lymph node metastasis. Oncotarget. 2014; 5:4426-37. https://doi.org/10.18632/oncotarget.2032.

11. Japp AG, Cruden NL, Barnes G, van Gemeren N, Mathews J, Adamson J, Johnston NR, Denvir MA, Megson IL, Flapan AD, Newby DE. Acute cardiovascular effects of apelin in humans: potential role in patients with chronic heart failure. Circulation. 2010; 121:1818-1827.

12. Ceylan-Isik AF, Kandadi MR, Xu X, Hua Y, Chicco AJ, Ren J, Nair S. Apelin administration ameliorates high fat diet-induced cardiac hypertrophy and contractile dysfunction. J Mol Cell Cardiol. 2013; 63:4-13.

13. Riazian M, Khorrami E, Alipoor E, Moradmand S, Yaseri M, Hosseinzadeh-Attar MJ. Assessment of Apelin Serum Levels in Persistent Atrial Fibrillation and Coronary Artery Disease. Am J Med Sci. 2016; 352:354-359.

14. Liu HT, Chen M, Yu J, Li WJ, Tao L, Li Y, Guo WY, Wang HC. Serum apelin level predicts the major adverse cardiac events in patients with ST elevation myocardial infarction receiving percutaneous coronary intervention. Medicine (Baltimore). 2015; 94:e449.

15. Zhou Y, Wang Y, Qiao S. Apelin: a potential marker of coronary artery stenosis and atherosclerotic plaque stability in ACS patients. Int Heart J. 2014; 55:204-212.

16. Kadoglou NP, Lampropoulos S, Kapelouzou A, Gkontopoulos A, Theofilogiannakos EK, Fotiadis G, Kottas G. Serum levels of apelin and ghrelin in patients with acute coronary syndromes and established coronary artery disease-KOZANI STUDY. Transl Res. 2010; 155:238246.

17. Mahar MA, Rainio A, Ilves M, Lindgren K, KarjaKoskenkari P, Taskinen P, Vuolteenaho O, Biancari F. Changes in natriuretic peptides, apelin and adrenomedullin after off-pump and on-pump coronary artery bypass surgery. J Cardiovasc Surg (Torino). 2008; 49:783-791.

18. Ye J, Ni P, Kang L, Xu B. Apelin and vascular endothelial 
growth factor are associated with mobilization of endothelial progenitor cells after acute myocardial infarction. J Biomed Res. 2012; 26:400-409.

19. Akcilar R, Yumun G, Bayat Z, Donbaloglu O, Erselcan $\mathrm{K}$, Ece E, Kokdasgil H, Genc O. Characterization of the apelin $-1860 \mathrm{~T}>\mathrm{C}$ polymorphism in Turkish coronary artery disease patients and healthy individuals. Int J Physiol Pathophysiol Pharmacol. 2015; 7:165-171.

20. Bilik MZ, Kaplan I, Yildiz A, Akil MA, Acet H, Yuksel M, Polat N, Aydin M, Oylumlu M, Ertas F, Kaya H, Alan S. Apelin Levels In Isolated Coronary Artery Ectasia. Korean Circ J. 2015; 45:386-390.

21. Grzywocz P, Mizia-Stec K, Wybraniec M, Chudek J. Adipokines and endothelial dysfunction in acute myocardial infarction and the risk of recurrent cardiovascular events. J Cardiovasc Med (Hagerstown). 2015; 16:37-44.

22. Pang H, Han B, Li ZY, Fu Q. Identification of molecular markers in patients with hypertensive heart disease accompanied with coronary artery disease. Genet Mol Res. 2015; 14:93-100.

23. Gupta MD, Girish MP, Shah D, Rain M, Mehta V, Tyagi S, Trehan V, Pasha Q. Biochemical and genetic role of apelin in essential hypertension and acute coronary syndrome. Int J Cardiol. 2016; 223:374-378.

24. Hinohara K, Nakajima T, Sasaoka T, Sawabe M, Lee BS, Ban JM, Park JE, Izumi T, Kimura A. Validation of the association between AGTRL1 polymorphism and coronary artery disease in the Japanese and Korean populations. J Hum Genet. 2009; 54:554-556.

25. Jin W, Su X, Xu M, Liu Y, Shi J, Lu L, Niu W. Interactive association of five candidate polymorphisms in Apelin/ APJ pathway with coronary artery disease among Chinese hypertensive patients. PLoS One. 2012; 7:e51123.

26. Wang $\mathrm{P}, \mathrm{Xu} \mathrm{C}$, Wang $\mathrm{C}, \mathrm{Wu} \mathrm{Y}$, Wang D, Chen S, Zhao Y, Wang X, Li S, Yang Q, Zeng Q, Tu X, Liao Y, et al. Association of SNP Rs9943582 in APLNR with Left Ventricle Systolic Dysfunction in Patients with Coronary Artery Disease in a Chinese Han GeneID Population. PLoS One. 2015; 10:e125926.

27. Kuklinska AM, Sobkowicz B, Sawicki R, Musial WJ, Waszkiewicz E, Bolinska S, Malyszko J. Apelin: a novel marker for the patients with first ST-elevation myocardial infarction. Heart Vessels. 2010; 25:363-367.

28. Tycinska AM, Sobkowicz B, Mroczko B, Sawicki R, Musial WJ, Dobrzycki S, Waszkiewicz E, Knapp MA, Szmitkowski M. The value of apelin-36 and brain natriuretic peptide measurements in patients with first STelevation myocardial infarction. Clin Chim Acta. 2010; 411:2014-2018.

29. Boal F, Timotin A, Roumegoux J, Alfarano C, Calise D, Anesia R, Parini A, Valet P, Tronchere H, Kunduzova O. Apelin-13 administration protects against ischaemia/ reperfusion-mediated apoptosis through the FoxO1 pathway in high-fat diet-induced obesity. Br J Pharmacol. 2016;
173:1850-1863

30. Sawane M, Kajiya K, Kidoya H, Takagi M, Muramatsu F, Takakura N. Apelin inhibits diet-induced obesity by enhancing lymphatic and blood vessel integrity. Diabetes. 2013; 62:1970-1980.

31. Boal F, Roumegoux J, Alfarano C, Timotin A, Calise D, Anesia R, Drougard A, Knauf C, Lagente C, Roncalli J, Desmoulin F, Tronchere H, Valet P, et al. Apelin regulates FoxO3 translocation to mediate cardioprotective responses to myocardial injury and obesity. Sci Rep. 2015; 5:16104.

32. De Mota N, Reaux-Le Goazigo A, El Messari S, Chartrel N, Roesch D, Dujardin C, Kordon C, Vaudry H, Moos F, Llorens-Cortes C. Apelin, a potent diuretic neuropeptide counteracting vasopressin actions through inhibition of vasopressin neuron activity and vasopressin release. Proc Natl Acad Sci U S A. 2004; 101:10464-10469.

33. Chandrasekaran B, Dar O, McDonagh T. The role of apelin in cardiovascular function and heart failure. Eur J Heart Fail. 2008; 10:725-732.

34. Gunter S, Solomon A, Tsang L, Woodiwiss AJ, Robinson C, Millen AM, Norton GR, Dessein PH. Apelin concentrations are associated with altered atherosclerotic plaque stability mediator levels and atherosclerosis in rheumatoid arthritis. Atherosclerosis. 2017; 256:75-81.

35. Reinehr T, Woelfle J, Roth CL. Lack of association between apelin, insulin resistance, cardiovascular risk factors, and obesity in children: a longitudinal analysis. Metabolism. 2011; 60:1349-1354.

36. Fujie S, Sato K, Miyamoto-Mikami E, Hasegawa N, Fujita S, Sanada K, Hamaoka T, Iemitsu M. Reduction of arterial stiffness by exercise training is associated with increasing plasma apelin level in middle-aged and older adults. PLoS One. 2014; 9:e93545.

37. McPherson R, Tybjaerg-Hansen A. Genetics of Coronary Artery Disease. Circ Res. 2016; 118:564-578.

38. Li G, Sun X, Zhao D, He L, Zheng L, Xue J, Wang B, Pan $\mathrm{H}$. A promoter polymorphism in APJ gene is significantly associated with blood pressure changes and hypertension risk in Chinese women. Oncotarget. 2016; 7:86257-65. https://doi.org/10.18632/oncotarget.13370.

39. Burdett S, Stewart LA, Tierney JF. Publication bias and meta-analyses: a practical example. Int J Technol Assess Health Care. 2003; 19:129-134.

40. Stroup DF, Berlin JA, Morton SC, Olkin I, Williamson GD, Rennie D, Moher D, Becker BJ, Sipe TA, Thacker SB. Meta-analysis of observational studies in epidemiology: a proposal for reporting. Meta-analysis Of Observational Studies in Epidemiology (MOOSE) group. JAMA. 2000; 283:2008-2012.

41. Phillips CV. Publication bias in situ. BMC Med Res Methodol. 2004; 4:20. 